\title{
Negative lymph node at station 108 is a strong predictor of overall survival in esophageal cancer
}

\author{
JINLING ZHANG ${ }^{1,2}$, XUEYUAN HENG ${ }^{2}$, YI LUO ${ }^{3}$, LUNING LI $^{2}$, HAIYAN ZHANG $^{2}$, \\ FENGYUAN $\mathrm{CHE}^{2}$ and BAOSHENG $\mathrm{LI}^{1}$
}

\author{
${ }^{1}$ Department of Radiation Oncology, Shandong Cancer Hospital, Shandong Academy of Medical Sciences, \\ Shandong University, Jinan, Shandong 250117; ${ }^{2}$ Department of Oncology, Linyi People's Hospital, Shandong University, \\ School of Medicine, Linyi, Shandong 276000, P.R. China; ${ }^{3}$ Department of Radiation Oncology, \\ University of Michigan, Ann Arbor, MI 48105, USA
}

Received August 14, 2017; Accepted April 6, 2018

DOI: $10.3892 / \mathrm{ol} .2018 .9456$

\begin{abstract}
A negative lymph node (NLN) may represent a stronger predictor for the overall survival (OS) rate of patients with esophageal squamous cell carcinoma (ESCC), when compared with a positive LN (PLN). The present study aimed to investigate which LN station, containing the NLN, was associated with OS rate. A retrospective review was conducted in 216 patients with ESCC and a forward stepwise Cox regression model analysis was used to assess the relationship between clinical parameters and OS rate. Patients were divided into subgroups according to the status of the LN at station 108. Survival analysis was performed using the Kaplan-Meier method. The ratio of albumin-to-globulin (AGR), and of lymphocytes to neutrophil granulocytes (LNR) in the subgroups were also investigated. Overall, 105p (the PLN number at station 105), 108p, 109p and 7p were confirmed to be risk factors for OS rate (all $\mathrm{P}<0.05$ ). Conversely, $108 \mathrm{n}$ (the NLN number at station 108) was identified as a protective factor for OS rate [hazard ratio (HR) $0.457, \mathrm{P}=0.001$ ]. Survival analysis demonstrated that patients with an NLN identified at the station 108 had an improved OS rate compared with those with a PLN identified at station $108(\mathrm{P}=0.006)$. Patients with only an NLN identified at station 108 had the best OS rate among all the sub-groups examined, and the AGR of this group of patients was higher than those of the other groups. The LN status at station 108 may indicate the prognosis of
\end{abstract}

Correspondence to: Dr Fengyuan Che, Department of Oncology, Linyi People's Hospital, Shandong University, School of Medicine, 27 Jiefang Road, Shandong 276000, P.R. China

E-mail: che1971@126.com

Dr Baosheng Li, Department of Radiation Oncology, Shandong Cancer Hospital, Shandong Academy of Medical Sciences, Shandong University, 440 Jiyan Road, Jinan, Shandong 250117, P.R. China

E-mail: baoshli1963@163.com

Key words: lymphatic metastasis, esophageal cancer, negative metastasis, prognosis, albumin to globin ratio patients with ESCC, and an NLN may reflect the reaction of the immune system to tumor metastasis in these patients.

\section{Introduction}

Esophageal squamous cell carcinoma (ESCC) is the principal pathological subtype of thoracic esophageal cancer reported in Asia in over the previous 30 years (1-3). Although comprehensive therapeutic strategies, including surgery, radiation therapy and chemotherapy, have been applied, the overall survival (OS) rate of ESCC remains poor (4). It has been widely recognized that lymph node metastasis (LNM) usually occurs in the early stages of disease, and serves an important role in the poor prognosis of patients with ESCC (5).

Previous studies have published the distribution pattern of LNM and investigated the influence of the surgical removal of the LN on the prognosis of ESCC $(6,7)$. The American Joint Committee on the Tumor Node Metastasis (TNM) system defined the $\mathrm{N}$-staging classification using the number of positive LN (PLN) collected during surgery (8). However, the number of negative LN (NLN) identified pathologically following surgery has been considered to be an even stronger predictor of OS rate in patients with ESCC (9). The reason may be that the number of NLN could reflect the extent and quality of the individual surgery, and be considered a critical factor in the prognosis of patients with ESCC. To the best of our knowledge, it is unclear which LN station containing the NLN served a key role in the prognosis of patients with ESCC; therefore, improving the presently available knowledge would be helpful for the design of treatment plans for postoperative radiotherapy. Research had proven that radiotherapy serves a key role in decreasing the probability of local tumor recurrence following surgery, and in prolonging OS rate; however, there was an issue, in that the design of treatment plans for radiotherapy was based on the metastasis pattern of PLN, without considering the effect of NLN on the prognosis of patients $(10,11)$. In other words, it was necessary to specify which LN station containing the NLN was associated with OS rate in patients with ESCC. Therefore, the present study aimed to investigate in which $\mathrm{LN}$ station the presence of NLN had a great impact on OS rate, for patients with middle and lower thoracic ESCC. 


\section{Patients and methods}

Patient population. A retrospective review was performed on the medical records of 216 patients with ESCC, who underwent esophagectomy and lymphadenectomy at Linyi People's Hospital, Shandong University (Linyi, China) between January 2009 and January 2013. The Institutional Review Board of Linyi People's Hospital approved the present study and all patients provided written informed consent. The criterion for patient selection were as follows: i) The patient received upper gastrointestinal endoscopy and upper gastrointestinal barium swallow, and the site of primary tumor was confirmed in the thoracic esophagus; ii) an ultrasound of the cervical region, computed tomography (CT) of chest and upper abdomen were performed to exclude distant metastasis of tumor; iii) the patient did not receive neo-adjuvant radiotherapy or chemotherapy prior to surgery; iv) the surgical specimen of the patient was identified as ESCC by two pathologists in the Department of Pathology in Linyi People's Hospital following surgery, and discussed uncertain samples until they reached an agreement; v) Tumor-free resection margin of the removed tissue were confirmed following surgery by a pathologist.

Treatment method. All patients underwent an extended esophagectomy with three-field or two-field LN dissection. Patients who underwent three-field LN dissection with tumor metastasis in the cervical region were considered based on an ultrasound or CT examination. The three-field LN dissections consisted of a collar neck incision, and a right thoracotomy and laparotomy, compared with the two-field LN dissection (including only a right thoracotomy and laparotomy). The dissection and labeling of LN stations was performed according to the system of the Japanese Society for Esophageal Diseases (12), as presented in Table I. The surgical specimens and LN marked with site labels were collected at the end of surgery, and were evaluated by two specialist pathologists from the Department of Pathology in Linyi People's Hospital (Shangdong, China). All the specimens and LN were fixed in $10 \%$ formalin for $24 \mathrm{~h}$ under room temperature and embedded in paraffin, and then subjected to $0.5 \%$ concentration of hematoxylin and $2 \%$ concentration of eosin staining at room temperature for $0.5 \mathrm{~h}$ after sectioning (5- $\mu \mathrm{m}$ thick slices). Metastasis in the LN was identified following a consensus from the two pathologists using a light microscope with magnification $\times 200$, and the numbers of PLN and NLN in each LN station were recorded. The pathological tumor stage of each case was evaluated according to the American Joint Committee on Cancer (AJCC) TNM classification (7th edition) (8). The post-operative treatment was selected according to the TNM stage of the tumor in each patient, and patients with an identical TNM stage received the same post-operative treatment. In another word, for example, patients with stage III of tumor were treated by chemo- and radiotherapy.

Follow-up. All patients underwent follow-up every 3 months for the first 2 years following surgical resection, and then every 6 months for the following 3 years. Upper gastrointestinal barium swallow, and CT or magnetic resonance imaging of the chest and upper abdomen, were used to evaluate the local recurrence and remote metastasis of the tumors. Patients who could not return to Linyi People's Hospital regularly were followed up by telephone. The final follow-up was conducted in January 2016. OS rate was evaluated as the time between surgery and patient mortality. The time of censoring was calculated from the date of surgery to the date of our last contact with the surviving patient.

Classification method of sub groups. Patients were initially divided into two groups, which included patients with PLN identified in the LN station 108, and patients with NLN identified in the LN station 108. Secondly, the patients were further divided into four groups, according to the status of the LN in the LN station 108, which can be described as follows: Group 1, patients whose PLN and NLN were identified in LN station 108 following surgery; Group 2, patients whose PLN were identified in LN station 108 following surgery; Group 3, patients whose NLN were identified in the LN station 108 following surgery; and Group 4, patients whose LN were not identified in the LN station 108 following surgery.

Ratio of albumin to globulin (AGR) and ratio of lymphocytes to neutrophils (LNR). Information regarding albumin, globulin, lymphocytes and neutrophils in the blood was collected from the medical records of each patient. Four time points were selected to collect the information at follow-up: The first time point was one week prior to surgery (AGR1 and LNR1); the second was one week after surgery (AGR2 and LNR2); the third was two weeks after surgery (AGR3 and LNR3); and the fourth was one month after surgery (AGR4 and LNR4). The ratio of AGR was calculated as the quantity of albumin divided by the quantity of globulin in the blood. Accordingly, the ratio of LNR was calculated using the number of lymphocytes divided by the number of neutrophils in the blood. The AGR and LNR at the four time points previously mentioned were calculated and used for subsequent analysis between patient subgroups. The differences in the ratios were investigated among the four groups prior to and following surgery.

Statistical analysis. The data in the present study are presented as the mean \pm standard deviation. The relationship between clinical parameters and the number of PLN or NLN were explored using the $\chi^{2}$ test and a forward stepwise Cox regression model analysis was further used to evaluate the associations between the above parameters and prognosis. Survival analysis was performed using Kaplan-Meier method and the differences of survival time between patient subgroups were investigated by log rank test. All the statistical analyses were computed using Stata/MP 13 (Stata Corp LP, College Station, TX, USA) and $\mathrm{P}<0.05$ was considered to indicate a statistically significant difference.

\section{Results}

Patient characteristics and OS rate. The number of censored case in this study was 24 (11.9\%), which was lower than the average level in clinical study (13). Excluding the patients who failed to attend for follow-up ( 24 cases), the medical information of the remaining 192 patients who completed follow-up was retrospectively reviewed. The characteristics of patients 
Table I. Terminology of the regional lymph nodes in esophageal cancer.

\begin{tabular}{ll}
\hline LN station no. & \multicolumn{1}{c}{ JSED (location) } \\
\hline 100 & Cervical compartment \\
101 & Paraesophageal nodes \\
102 & Deep cervical nodes \\
103 & Retropharyngeal lymph nodes \\
104 & Supraclavicular lymph nodes \\
105 & Upper thoracic paraesophageal \\
106 & Thoracic paratracheal lymph nodes \\
107 & Bifurcational \\
108 & Middle thoracic paraesophageal \\
109 & Main bronchus (R, L) \\
110 & Lower thoracic paraesophageal \\
111 & Diaphragmatic lymph nodes \\
1 & Right cardiac nodes \\
2 & Left cardiac nodes \\
3 & Nodes along the lesser curvature \\
4 & Nodes along the greater curvature \\
5 & Suprapyloric nodes \\
6 & Infrapyloric nodes \\
7 & Left gastric artery \\
\hline
\end{tabular}

LN, lymph node; JSED, Japanese Society for Esophageal Diseases; $\mathrm{R}$, right; L, left.

are shown in Table II. LNM were identified in 100 of the 192 patients, and the mean number of PLN was 0.874 (SD, 1.046; range, 1-5; median, 1) while the mean number of NLN was 3.168 (SD, 1.319; range, 1-7; median, 3). In the group of patients without LNM, the mean number of NLN was 3.195 (SD, 1.297; range, 1-6; median, 3). The mean follow-up time was 27.2 months (SD, 18.8; range, 3-73 months; median, 24 months). On the basis of the analysis for the entire cohort (192 patients), the 1, 3 and 5-year survival rates were 72.9, 38.9 and $23.8 \%$, respectively.

Association between the LN station containing the NLN and patient survival. In the univariate analysis of the association between patient clinicopathological features and the LN status, the tumor length, tumor differentiation, depth of tumor invasion, tumor site, nerve invasion and lymph vessel invasion were all risk factors for the number of PLN, while only lymph vessel invasion was identified as a risk factor for the number of NLN (Table II). All possible risk factors were subsequently put into a stepwise Cox analysis model, and 108p (the PLN number in LN station 108) and 109p (the PLN number in LN station 109) were confirmed as independent prognostic factors for OS rate (Table III). Conversely, 108n (the NLN number in LN station 108) was highlighted as a protective factor for OS rate. In the analysis of the association between $108 \mathrm{n}$ and tumor relapse, the former was proven to have statistical effect against the latter (Table IV).

LN status of LN station 108 and OS rate of patients. The results of log-rank tests revealed that the subgroup of patients with
NLN identified in LN station 108 had an improved OS rate than those with PLN identified at LN station $108(\mathrm{P}=0.006)$. The mean OS times of the two sub-groups were 30.0 months (SD, 18.197; range, 3-73 months; median, 28 months) and 27.436 months (SD, 19.129; range, 3-73 months; median, 24 months). The 1-, 3- and 5-year survival rates for the patients with NLN identified in LN station 108 were 80.4, 46.9 and $27.5 \%$, respectively, while the rates for the patients with PLN identified in LN station 108 were 62.5, 27.0 and 21.2\%, respectively (Fig. 1).

Further comparative analysis confirmed the presence of significant differences in OS rate among the four groups. Group 3 had the best outcome with a mean OS time of 34.208 months when compared with the other groups (Group 3 vs. Group 1: $\mathrm{P}<0.001$; Group 3 vs. Group 2: $\mathrm{P}<0.001$; Group 3 vs. Group 4: $\mathrm{P}=0.001)$. Group 2 had the worst outcome with a mean OS time of 10.375 months of any group (Group 2 vs. Group 1: $\mathrm{P}=0.018$; Group 2 vs. Group 3: $\mathrm{P}<0.001$; Group 2 vs. Group 4: $\mathrm{P}=0.007$.). However, there was no significant difference between Group 4 and Group 1 of which the OS times were 23.847 and 22.389 months, respectively $(\mathrm{P}=0.667$; Fig. 2). Group 2 was the group of patients with only PLN identified in LN station 108 after surgery and was used as the control.

Change in AGR and LNR in the subgroups following surgery. The AGR and LNR of patients decreased following surgery in all except Group 2, for which insufficient information regarding the AGR and LNR was obtained (data not shown). At the initial diagnosis of the disease, the number of AGR was similar amongst the three groups. However, following the operation, the number of AGR in Group 3 was higher than that of any other group until three months later. Similarly, the number of LNR were similar to one another amongst the three groups prior to the operation, however, the number of LNR in Groups 3 was higher compared with the other two groups until two months later (Fig. 3).

\section{Discussion}

Previous studies have demonstrated that a greater number of NLN identified during surgery indicated an improved OS rate $(9,14)$. The underlying mechanism of the important role of NLN in prognosis remains uncertain. Interpreting this has led to two possible hypotheses $(15,16)$.

The first hypothesis is 'stage migration'. The extent of lymphadenectomy is insufficient if only PLN are removed in ESCC. The $\mathrm{N}$ stage of a tumor can be precisely evaluated only when sufficient NLN and PLN are removed via lymphadenectomy; otherwise, late-stage ESCC may be erroneously classified as early-stage ESCC. The second hypothesis is that the collection of more NLN during surgery may decrease the false-negative error rate in the pathological examination of LN. Researchers have previously demonstrated that tumor metastasis may be present in NLN, as determined by immunohistochemical staining methods, which may explain the aforementioned false-negative error rate $(17,18)$. Increased identification of NLN by a pathologist may decrease the possibility of an incorrect evaluation of the $\mathrm{N}$ stage of a tumor. 
Table II. Clinical and pathological variables of the patients with pathologically confirmed positive lymph node.

\begin{tabular}{|c|c|c|c|c|c|}
\hline Characteristics & $\begin{array}{c}\text { Total } \\
\text { number of LN }\end{array}$ & $\begin{array}{c}\text { Number } \\
\text { of positive LN }\end{array}$ & P-value & $\begin{array}{c}\text { Number } \\
\text { of negative LN }\end{array}$ & P-value \\
\hline \multicolumn{6}{|l|}{ Age (years) } \\
\hline$<60$ & 970 & 117 & \multirow[t]{2}{*}{0.630} & 853 & \multirow[t]{2}{*}{0.893} \\
\hline$\geq 60$ & 1742 & 198 & & 1544 & \\
\hline \multicolumn{6}{|l|}{ Sex } \\
\hline Men & 2447 & 294 & \multirow[t]{2}{*}{0.075} & 2153 & \multirow[t]{2}{*}{0.627} \\
\hline Women & 265 & 21 & & 244 & \\
\hline \multicolumn{6}{|c|}{ Length of tumor (cm) } \\
\hline$\leq 4$ & 1201 & 94 & \multirow[t]{3}{*}{$<0.001$} & 1107 & \multirow[t]{3}{*}{0.429} \\
\hline $4-6$ & 775 & 118 & & 657 & \\
\hline$\geq 6$ & 736 & 95 & & 641 & \\
\hline \multicolumn{6}{|l|}{ Tumor site } \\
\hline Upper & 92 & 2 & \multirow[t]{3}{*}{$<0.001$} & 90 & \multirow[t]{3}{*}{0.463} \\
\hline Middle & 1653 & 165 & & 1488 & \\
\hline Lower & 967 & 148 & & 819 & \\
\hline \multicolumn{6}{|l|}{ Differentiation } \\
\hline Well & 778 & 47 & \multirow[t]{3}{*}{$<0.001$} & 731 & \multirow[t]{3}{*}{0.419} \\
\hline Moderate & 1569 & 210 & & 1359 & \\
\hline Poor & 356 & 47 & & 309 & \\
\hline \multicolumn{6}{|c|}{ Depth of tumor invasion } \\
\hline $\mathrm{T} 1-\mathrm{T} 2$ & 2091 & 35 & \multirow[t]{2}{*}{$<0.001$} & 2056 & \multirow[t]{2}{*}{0.347} \\
\hline $\mathrm{T} 3-\mathrm{T} 4$ & 621 & 47 & & 574 & \\
\hline \multicolumn{6}{|l|}{ Nerve invasion } \\
\hline Yes & 119 & 31 & \multirow[t]{2}{*}{$<0.001$} & 88 & \multirow[t]{2}{*}{0.195} \\
\hline No & 2593 & 284 & & 2309 & \\
\hline \multicolumn{6}{|c|}{ Lymph vessel invasion } \\
\hline Yes & 164 & 52 & \multirow[t]{2}{*}{$<0.001$} & 112 & \multirow[t]{2}{*}{0.030} \\
\hline No & 2548 & 263 & & 2285 & \\
\hline \multicolumn{6}{|l|}{ LN dissention } \\
\hline Two-field & 1389 & 178 & \multirow[t]{2}{*}{0.006} & 1211 & \multirow[t]{2}{*}{0.461} \\
\hline Three-field & 1323 & 121 & & 1202 & \\
\hline \multicolumn{6}{|c|}{ Postoperative radiochemotherapy } \\
\hline Yes & 1246 & 156 & \multirow[t]{2}{*}{0.228} & 1090 & 0.736 \\
\hline No & 1466 & 159 & & 1307 & \\
\hline
\end{tabular}

LN, lymph node; LNM, lymph node metastases; calculated with a $\chi^{2}$-square test.

Previous studies have stated that the site of LNM is a more important prognostic factor than the number of LNM, and it was necessary to clarify which LN station containing the NLN would most significantly influence the outcome of patients $(19,20)$.

The present study identified that the presence of NLN in LN station 108 could significantly influence the outcomes of patients, on the basis of multivariate Cox regression analysis and survival analysis. Furthermore, patients had a better prognosis if only NLN were identified in LN station 108, and these patients were primarily in the early $\mathrm{N}$ stage, which supports the 'stage migration' theory. The present study also identified that the LN stations 107 and 7 were high-efficiency LN stations containing NLN in patients with ESCC at an early $\mathrm{N}$ stage, which was in accordance with our previously published results (21).

As the LN station 108 was categorically removed in the lymphadenectomy, regardless of whether three-field or two-field LN dissection was performed, and was used to assess the prognosis of patients and/or the extent of lymphadenectomy. The present study demonstrated that patients with only NLN identified in LN station 108 had an improved prognosis when compared with the other subgroups, indicating it should be seriously considered whether postoperative radiation therapy or chemotherapy treatment is appropriate for this group of patients. On the other hand, 
Table III. Association between the total number of positive and negative of LN and overall survival.

\begin{tabular}{lcccrrr}
\hline Factor & Hazard ratio & Standard error & Z coefficient & P-value & [95\% conf. & Interval] \\
\hline $108 \mathrm{P}$ & 2.978 & 0.710 & 4.58 & $<0.001$ & 1.866 \\
$108 \mathrm{~N}$ & 0.475 & 0.102 & -3.47 & 0.001 & 0.312 \\
$108 \mathrm{P}+105 \mathrm{P}$ & 5.606 & 4.226 & 2.29 & 0.022 & 1.279 & 24.750 \\
$105 \mathrm{P}$ & 14.165 & 11.427 & 3.29 & 0.001 & 2.914 \\
$109 \mathrm{P}$ & 10.756 & 11.545 & 2.21 & 0.027 & 1.312 \\
$7 \mathrm{P}$ & 1.771 & 0.436 & 2.32 & 0.020 & 1.094 \\
Diff & 1.322 & 0.199 & 1.86 & 0.064 & 0.984 & 2.868 \\
& & & & 1.776
\end{tabular}

LN, lymph node; P, positive LN; N, negative LN; Diff, differentiation of tumor.

Table IV. Association between the total number of positive and negative of LN and tumor relapse.

\begin{tabular}{|c|c|c|c|c|c|c|}
\hline Factor & Hazard ratio & Standard error & $\mathrm{Z}$ coefficient & P-value & [95\% conf. & Interval] \\
\hline $108 \mathrm{P}$ & 2.506 & 0.777 & 2.96 & 0.003 & 1.364 & 4.602 \\
\hline $105 \mathrm{P}$ & 9.768 & 11.002 & 2.02 & 0.043 & 1.074 & 88.826 \\
\hline $109 \mathrm{P}$ & 13.539 & 14.754 & 2.39 & 0.017 & 1.599 & 114.601 \\
\hline $108 \mathrm{~N}$ & 0.501 & 0.139 & -2.48 & 0.013 & 0.290 & 0.864 \\
\hline $107 \mathrm{~N}$ & 0.546 & 0.139 & -2.37 & 0.018 & 0.331 & 0.900 \\
\hline Diff & 1.559 & 0.312 & 2.21 & 0.027 & 1.052 & 2.309 \\
\hline
\end{tabular}

LN, lymph node; P, positive LN; N, negative LN; Diff, differentiation of tumor.

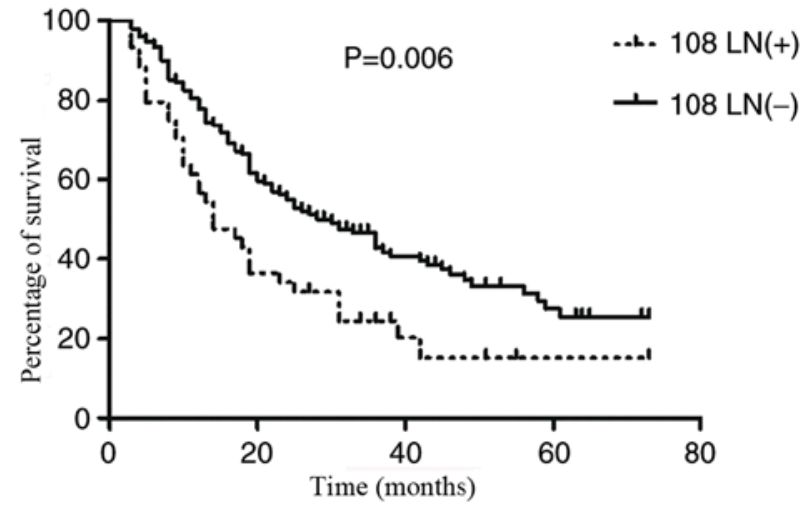

Figure 1. The comparison of OS rate between patients with NLN identified in LN station 108 and those with PLN identified in LN station 108. The difference of OS rate between patients with NLN identified in LN station 108 and those with PLN identified in LN station 108 were statistically significant, $(\mathrm{P}=0.006)$. OS, overall survival; NLN, negative nymph node; LN, lymph node; PLN, positive lymph node; + , positive; -, negative.

since the extent of lymphadenectomy remains controversial at present, it was worth exploring whether this group of patients could benefit from surgery wherein more LNs are removed via lymphadenectomy $(22,23)$.

Although the mechanism underlying the prognostic role of NLN in LN station 108 is complex, it could be interpreted from interactions between the patient immune system and tumor metastasis. LN station 108 is the closest LN station to the esophagus, and may be the first station, or the foothold, for

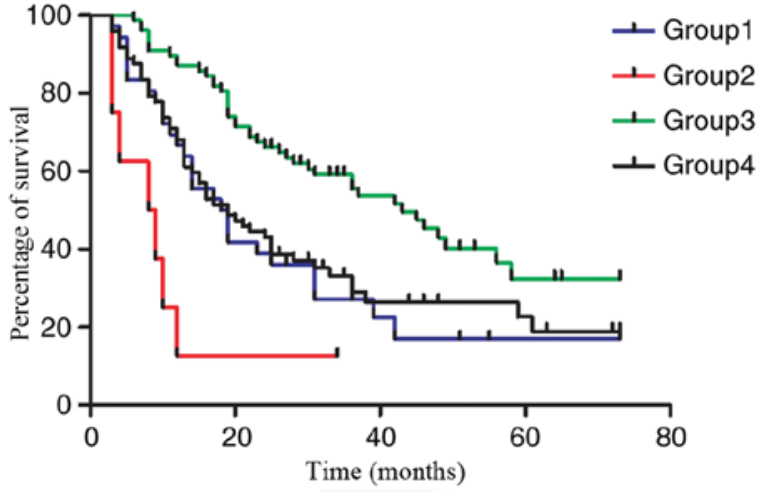

Figure 2. The comparison of OS rate among the subgroups of patients. A significant difference in the OS rate was identified among the four groups $(\mathrm{P}<0.001)$. Furthermore, significant differences were confirmed between each subgroup except the comparison between Groups 4 and $1(\mathrm{P}>0.05)$. The other comparison were as follows: Group 1 vs. 2, $\mathrm{P}=0.018$; Group 1 vs. 3 , $\mathrm{P}<0.001$; Group 2 vs. $3, \mathrm{P}<0.001$; Group 2 vs. $4, \mathrm{P}=0.007$; and Group 3 vs. 4 , $\mathrm{P}=0.001$. OS, overall survival.

tumor metastasis (12). Patients with only NLN identified in LN station 108 may be in a stable condition due to immune system resistance to tumor metastasis. Dynamic investigation of the change in AGR and LNR in the present study supported this assumption. AGR and LNR were higher in patients with NLN identified in LN station 108 than those with PLN identified in LN station 108, and the AGR was significantly higher in patients with only NLN identified in LN station 108 compared with those in the other groups. This was consistent with prior reports 

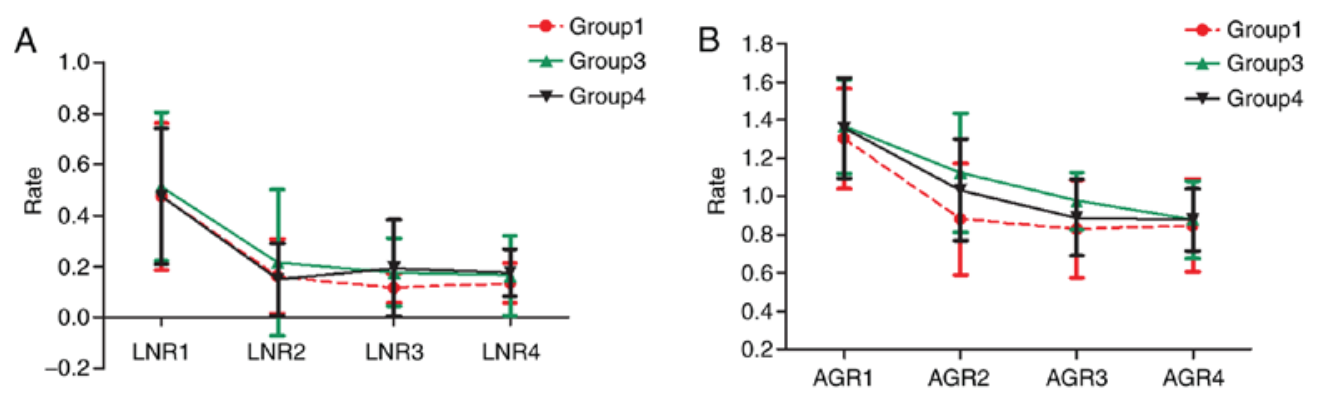

Figure 3. The change of LNR and AGR following surgery in subgroups. The rate of LNRs and AGRs were collected at four time points. LNR1 and AGR1 were obtained one week prior to surgical resection while LNR2 and AGR2, LNR3 and AGR3, and LNR4 and AGR4 were obtained at one week, two weeks and one month following surgery, respectively. (A) The LNR in all subgroups was decreased following surgery; however, the LNR in Group 4 and Group 3 were higher than Group 1. (B) The AGR also decreased after operation in all subgroups, but the level of AGR in Group 3 was consistently higher than those in other subgroups at the four examined time points. AGR, Ratio of albumin to globulin; LNR, ratio of lymphocytes to neutrophils.

that AGR and LNR have a close association with the prognosis of patients (24-26). Based on the results of the present study, the AGR may be a more reliable predictor of outcomes inpatients, compared with the LNR. The reason for this may be that AGR may provide a true reflection of the condition of a patient that the AGR provides the combined information of immune and nutritional situation of patients $(27,28)$. According to previous studies, AGR was considered to be closely associated with the patient immune system, and may be used as an indicator of the immune condition of patients in the future $(29,30)$. Based on the results of other reports, the change in LNR could reflect the T-cell-dependent immune response to a tumor $(31,32)$. However, one previous study produced the opposite results (33). The reason for this might be that LNR is less stable than AGR, which was confirmed in the present study. Based on the aforementioned results the LN status of LN station 108 has the potential to be used as an indicator for the selection of postoperative treatment. Chemo-radiotherapy would not be recommended for patients with only NLN identified in LN station 108, compared with the recommended treatment based on the tumor stage.

In the univariate analysis performed in the present study, the length of the tumor, tumor differentiation, depth of tumor invasion, tumor site, nerve invasion and lymph vessel invasion were risk factors for tumor metastasis, which was consistent with previous results (34). However, only the parameter of lymph vessel invasion influenced the number of NLN, which supported the interpretation, that tumor metastasis usually occurs through the lymph vessels in ESCC. Multivariate analysis revealed that the presence of negative $\mathrm{LN}$ in $\mathrm{LN}$ station 108 served a key role in tumor relapse and patient outcome, which supported the survival analysis results of the present study. The 1-, 3- and 5-year survival rates of patients were similar to those in reports published previously, and therefore, the selection bias of patients could be ignored in this study (35).

There were several limitations to this study. First, this study was not an example of large-scale research; however, the inclusion criterion was strict and the pathological type was limited to the thoracic squamous cell carcinoma in order to exclude confounders. Furthermore, the statistical results were cross-validated using several methods to avoid statistical bias. The present study has been completed according to the research plan supervised by the Linyi People's Hospital ethics committee (Linyi, China); however, another larger-scale study is currently being conducted. Additionally, the results of the present study could be repeated based on the preliminary analysis (data not shown), and due to the size limitation of the cohort, it was impossible to further investigate the influence of NLN on prognosis in the sub-groups classified by T stage or the type of LN dissection. As the number of patients with only PLN identified in the LN station 108 was small, classified as group 2 in this study, it was difficult to dynamically explore the LNR and AGR change patterns in this group. Secondly, the inclusion criteria of the patients did not set a threshold on the LN number removed in the surgery in this study. However, in the present study, the majority of cases experienced systematic lymphectomy. Furthermore, results without an artificial threshold for the LN number are more valuable for popularized application in the future. Thirdly, the site of $108 \mathrm{LN}$ station was adjacent to the $105 \mathrm{LN}$ station in anatomy, and it was even difficult for experienced surgeons to differentiate where the swollen and syncretic positive LN where located in some cases (36). To combat this, the present study labeled the positive LN in this situation as $108 \mathrm{P}+105 \mathrm{P}$.

To conclude, the results of this study demonstrated that the presence of NLN in LN station 108 could be used as a predictor of prognosis; it may also be used as an indicator to recommend the extent of $\mathrm{LN}$ dissection. A prospective study is required to explore whether the presence of NLN in LN station 108 may be used as an indicator for the selection of post-operative treatment, compared with the present method instructed by the TNM system, which neglected the reported impact of negative $\mathrm{LN}$ on the prognosis of patients. Although the present study preliminarily identified the potential underlying mechanisms and the key role of NLN in LN station 108 in prognosis, further research is still required in order to explore and validate these mechanisms. Our further test program includes two additional studies. The first one aims to demonstrate that the negative $\mathrm{LN}$ inside thoracic cavity serves an important role in the prognosis of patients using a larger scale of patients (which at the time of publication is ongoing, data not shown). The second study aims to explore whether the status of LN in station 108 serves a key role in patients with ESCC in a perspective study, and whether it may be used a marker to indicate the extent and efficacy of lymphadenectomy.

\section{Acknowledgements}

Not applicable. 


\section{Funding}

The present study was supported by Shandong Provincial Medical and Health Development Plan (grant no. 2013WSA13018, Dr Jinling Zhang), the Natural Science Foundation of Shandong Province (grant no. ZR2013HL018 and ZR2014HL062, Dr Jinling Zhang).

\section{Availability of data and materials}

The datasets used and/or analyzed during the present study are available from the corresponding author on reasonable request.

\section{Authors' contributions}

BL, FC, YL, JZ and HZ designed the study. XH and LL collected the information of patients and analyzed the data. JZ and $\mathrm{HZ}$ wrote the paper. FC, YL and BL reviewed and edited the manuscript. All authors read and approved the manuscript.

\section{Ethics approval and consent to participate}

All procedures used in the present study involving human participants were in accordance with the ethical standards of the institutional research committee, and with the 1964 Helsinki declaration and its later amendment or comparable ethical standards. The present study was approved by the Ethics Review Board of Linyi People's Hospital (Shangdong, China) and written informed consent was collected from each patient.

\section{Patient consent for publication}

Informed consent was obtained from all participants included in the study.

\section{Competing interests}

The authors declare that they have no competing interests.

\section{References}

1. Li B, Chen H, Xiang J, Zhang Y, Kong Y, Garfield DH and Li H: Prevalence of lymph node metastases in superficial esophageal squamous cell carcinoma. J Thorac Cardiovasc Surg 146: 1198-1203, 2013.

2. Merkow RP, Bilimoria KY, Keswani RN, Chung J, Sherman KL, Knab LM, Posner MC and Bentrem DJ: Treatment trends, risk of lymph node metastasis, and outcomes for localized esophageal cancer. J Natl Cancer Inst 106: pii: dju133, 2014.

3. McCormack VA, Menya D, Munishi MO, Dzamalala C, Gasmelseed N, Leon Roux M, Assefa M, Osano O, Watts M, Mwasamwaja AO, et al: Informing etiologic research priorities for squamous cell esophageal cancer in Africa: A review of setting-specific exposures to known and putative risk factors. Int J Cancer 140: 259-271, 2017.

4. Du D, Song T, Liang X, Fang M and Wu S: Concurrent chemoradiotherapy with elective lymph node irradiation for esophageal cancer: A systemic review and pooled analysis of the literature. Dis Esophagus 30: 1-9, 2017.

5. Liu J, Liu Q, Wang Y, Xia Z and Zhao G: Nodal skip metastasis is associated with a relatively poor prognosis in thoracic esophageal squamous cell carcinoma. Eur J Surg Oncol 42: 1202-1205, 2016

6. Dubecz A, Kern M, Solymosi N, Schweigert M and Stein HJ: Predictors of lymph node metastasis in surgically resected T1 esophageal cancer. Ann Thorac Surg 99: 1879-1886, 2015.
7. Ren X, Zhao Z, Huang W, Liu H, Dong C and Li Y: Analysis of the characteristics and factors influencing lymph node metastasis in thoracic esophageal carcinoma and cancer of the gastric cardia. Hepatogastroenterology 62: 73-76, 2015.

8. Zhang D, Zheng Y, Wang Z, Huang Q, Cao X, Wang F and Liu S: Comparison of the 7th and proposed 8th editions of the AJCC/UICC TNM staging system for esophageal squamous cell carcinoma underwent radical surgery. Eur J Surg Oncol 43: 1949-1955, 2017.

9. Baba Y, Watanabe M, Shigaki H, Iwagami S, Ishimoto T, Iwatsuki $\mathrm{M}$ and $\mathrm{Baba} \mathrm{H}$ : Negative lymph-node count is associated with survival in patients with resected esophageal squamous cell carcinoma. Surgery 153: 234-241, 2013.

10. Kim KH, Chang JS, Cha JH, Lee IJ, Kim DJ, Cho BC, Park KR and Lee CG: Optimal adjuvant treatment for curatively resected thoracic esophageal squamous cell carcinoma: A radiotherapy perspective. Cancer Res Treat 49: 168-177, 2017.

11. Wang S, Wang Z, Yang Z, Liu Y, Liu X, Shang B and Jiang WP: Postoperative radiotherapy improves survival in stage pT2NOM0 esophageal squamous cell carcinoma with high risk of poor prognosis. Ann Surg Oncol 23: 265-272, 2016.

12. Kajiyama Y: New Japanese classification of esophageal cancer (11th Edition). Gan To Kagaku Ryoho 43: 1049-1052, 2016 (In Japanese).

13. Cheng J, Kong L, Huang W, Li B, Li H, Wang Z, Zhang J, Zhou T and Sun H: Explore the radiotherapeutic clinical target volume delineation for thoracic esophageal squamous cell carcinoma from the pattern of lymphatic metastases. J Thorac Oncol 8: 359-365, 2013.

14. Greenstein AJ, Litle VR, Swanson SJ, Divino CM, Packer S and Wisnivesky JP: Effect of the number of lymph nodes sampled on postoperative survival of lymph node-negative esophageal cancer. Cancer 112: 1239-1246, 2008.

15. Zhu Z, Chen H, Yu W, Fu X, Xiang J, Li H, Zhang Y, Sun M, Wei Q, Zhao W and Zhao K: Number of negative lymph nodes is associated with survival in thoracic esophageal squamous cell carcinoma patients undergoing three-field lymphadenectomy. Ann Surg Oncol 21: 2857-2863, 2014.

16. Hsu PK, Huang CS, Wang BY, Wu YC, Chou TY and Hsu WH: The prognostic value of the number of negative lymph nodes in esophageal cancer patients after transthoracic resection. Ann Thorac Surg 96: 995-1001, 2013.

17. Luketich JD, Kassis ES, Shriver SP, Nguyen NT, Schauer PR, Weigel TL, Yousem SA and Siegfried JM: Detection of micrometastases in histologically negative lymph nodes in esophageal cancer. Ann Thorac Surg 66: 1715-1718, 1998.

18. Komukai S, Nishimaki T, Watanabe H, Ajioka Y, Suzuki T and Hatakeyama K: Significance of immunohistochemically demonstrated micrometastases to lymph nodes in esophageal cancer with histologically negative nodes. Surgery 127: 40-46, 2000.

19. de Meer SG, Dauwan M, de Keizer B, Valk GD, Borel Rinkes IH and Vriens MR: Not the number but the location of lymph nodes matters for recurrence rate and disease-free survival in patients with differentiated thyroid cancer. World J Surg 36: 1262-1267, 2012.

20. Tanaka H, Ohira M, Kubo N, Muguruma K, Yamashita Y, Sawada T and Hirakawa K: Association of location of lymph node metastases with postoperative recurrence of esophageal squamous cell carcinoma. Anticancer Res 32: 3421-3426, 2012.

21. Yang HX, Xu Y, Fu JH, Wang JY, Lin P and Rong TH: An evaluation of the number of lymph nodes examined and survival for node-negative esophageal carcinoma: Data from China. Ann Surg Oncol 17: 1901-1911, 2010.

22. Lagergren J, Mattsson F, Zylstra J, Chang F, Gossage J, Mason R, Lagergren P and Davies A: Extent of lymphadenectomy and prognosis after esophageal cancer surgery. JAMA Surg 151: 32-39, 2016.

23. van der Schaaf M, Johar A, Wijnhoven B, Lagergren P and Lagergren J: Extent of lymph node removal during esophageal cancer surgery and survival. J Natl Cancer Inst 107: pii: djv043, 2015.

24. Yodying H, Matsuda A, Miyashita M, Matsumoto S, Sakurazawa N, Yamada M and Uchida E: Prognostic significance of neutrophil-to-lymphocyte ratio and platelet-to-lymphocyte ratio in oncologic outcomes of esophageal cancer: A systematic review and meta-analysis. Ann Surg Oncol 23: 646-654, 2016.

25. Sharaiha RZ, Halazun KJ, Mirza F, Port JL, Lee PC, Neugut AI, Altorki NK and Abrams JA: Elevated preoperative neutrophil: Lymphocyte ratio as a predictor of postoperative disease recurrence in esophageal cancer. Ann Surg Oncol 18: 3362-3369, 2011. 
26. Zhang F, Sun P, Wang ZQ, Wang de S, Wang Y, Zhang DS, Wang FH, Fu JH, Xu RH and Li YH: Low preoperative albumin-globulin score predicts favorable survival in esophageal squamous cell carcinoma. Oncotarget 7: 30550-30560, 2016.

27. Fukushima H, Kobayashi M, Kawano $K$ and Morimoto S: Prognostic value of albumin/globulin ratio in patients with upper tract urothelial carcinoma patients treated with radical nephroureterectomy. Anticancer Res 38: 2329-2334, 2018.

28. He J, Pan H, Liang W, Xiao D, Chen X, Guo M and He J Prognostic effect of Albumin-to-Globulin ratio in patients with solid tumors: A systematic review and meta-analysis. J Cancer 8: 4002-4010, 2017.

29. Suh B, Park S, Shin DW, Yun JM, Keam B, Yang HK, Ahn E, Lee H, Park JH and Cho B: Low albumin-to-globulin ratio associated with cancer incidence and mortality in generally healthy adults. Ann Oncol 25: 2260-2266, 2014.

30. Shibutani M, Maeda K, Nagahara H, Ohtani H, Iseki Y, Ikeya T, Sugano K and Hirakawa K: The pretreatment albumin to globulin ratio predicts chemotherapeutic outcomes in patients with unresectable metastatic colorectal cancer. BMC Cancer 15: 347, 2015

31. Ji WH, Jiang YH, Ji YL, Li B and Mao WM: Prechemotherapy neutrophil:lymphocyte ratio is superior to the platelet: Lymphocyte ratio as a prognostic indicator for locally advanced esophageal squamous cell cancer treated with neoadjuvant chemotherapy. Dis Esophagus 29: 403-411, 2016.
32. Yutong H, Xiaoli X, Shumei L, Shan S, Di L and Baoen S: Increased neutrophil-lymphocyte ratio is a poor prognostic factor in patients with esophageal cancer in a high incidence area in China. Arch Med Res 46: 557-563, 2015.

33. Xie X, Luo KJ, Hu Y, Wang JY and Chen J: Prognostic value of preoperative platelet-lymphocyte and neutrophil-lymphocyte ratio in patients undergoing surgery for esophageal squamous cell cancer. Dis Esophagus 29: 79-85, 2016.

34. Matsuda S, Tsubosa Y, Niihara M, Sato H, Takebayashi K, Kawamorita K, Mori K, Tsushima T, Yasui H, Takeuchi H and Kitagawa Y: Distribution of lymph node metastasis and clinical validity of gastric tube reconstruction in lower thoracic esophageal squamous cell carcinoma with gastric invasion. Ann Surg Oncol 22: 617-623, 2015.

35. Wilke TJ, Bhirud AR and Lin C: A review of the impact of preoperative chemoradiotherapy on outcome and postoperative complications in esophageal cancer patients. Am J Clin Oncol 38: 415-421, 2015.

36. Yuasa Y, Seike J, Yoshida T, Takechi H, Yamai H, Yamamoto Y, Furukita Y, Goto M, Minato T, Nishino T, et al: Sentinel lymph node biopsy using intraoperative indocyanine green fluorescence imaging navigated with preoperative CT lymphography for superficial esophageal cancer. Ann Surg Oncol 19: 486-493, 2012. 